\title{
DESIGNING A SHIP COURSE CONTROLLER BY APPLYING THE ADAPTIVE BACKSTEPPING METHOD
}

\author{
ANNA WITKOWSKA, ROMAN ŚMIERZCHALSKI \\ Faculty of Electrical and Control Engineering \\ Gdańsk University of Technology, G. Narutowicza 11/12, 80-233 Gdańsk, Poland \\ e-mail: \{awitkowska, romsmier\}@ely.pg.gda.pl
}

\begin{abstract}
The article discusses the problem of designing a proper and efficient adaptive course-keeping control system for a seagoing ship based on the adaptive backstepping method. The proposed controller in the design stage takes into account the dynamic properties of the steering gear and the full nonlinear static maneuvering characteristic. The adjustable parameters of the achieved nonlinear control structure were tuned up by using the genetic algorithm in order to optimize the system performance. A realistic full-scale simulation model of the B-481 type vessel including wave and wind effects was applied to simulate the control algorithm by using time domain analysis.
\end{abstract}

Keywords: autopilot, adaptive control, nonlinear control, backstepping, genetic algorithm.

\section{Introduction}

Numerous investigations oriented toward the design of integrated ship control systems have been performed recently. Despite a significant improvement in automation, course control is still an active field of research. Nowadays, in marine systems it is necessary, when applying nonlinear adaptive techniques, to consider structural and model parametric uncertainties. This is due to the characteristics of a ship's big inertia, nonlinearity, perturbations of the parameters and random external disturbances.

In the literature, numerous attempts have been made to overcome these difficulties. The proposed methods include: neural network control (Richter and Burns, 1993; Simensen, 1995), fuzzy control (Zhang and Jia, 2000), forecast control (Du et al., 2004), and genetic and evolutionary algorithms (Karr, 1991; Alfarocid et al., 2005; McGookin et al., 2000). The control algorithms presented in the literature for the course changing process are mainly based on a dynamic ship model. They include linear-quadratic techniques (Zwierzewicz, 2004), $H_{\infty}$ control (Grimble et al., 1993; Messer and Grimble, 1993), feedback linearization control, disturbance rejection control (Han, 2002; Chen et al., 2008; Han et al., 2009), sliding mode control (Tomera, 2010; Ruan, 2006), and backstepping control.

These methods give solutions which are not fully satisfying. For example, there is chattering in sliding mode control. What is more, a high precision model is required in $H_{\infty}$ control, generalized forecast control, feedback linearization and the backstepping method. Nowadays, autopilots which are installed on ships usually use the algorithms of the PID controller, because of their simplicity, reliability and easiness of construction. Although some progress has been made in that method (fuzzy PD, ADRC, PD-backstepping), some disadvantages are still exposed. In fuzzy control, it is usually difficult to determine and optimize the control rules. It is always a hard work to optimize the parameters of a disturbance rejection controller (ADRC) because of a large number of parameters.

There is a main concept of the backstepping method when designing a globally stable control system for a class of nonlinear systems having a cascade structure. The construction for both the feedback control law and the accompanying Lyapunov function for systems with unknown parameters is a systematic procedure. This is an important property of the backstepping method. An overview of the first works published in the initial period of the development of the backstepping method has been presented by Kokotovic and Arcak (2001) as well as Fossen and Strand (1999). One of the most important works describing the backstepping method was published by Krstic et al. (1995). In marine technology, 
the presented method was used in ship control systems to secure course stabilization (Do et al., 2004), position and velocity stabilization or position and course stabilization (Pettersen and Nijmeijer, 2004), and to effectively control ships on a desired track (Bibuli et al., 2007; Almeida et al., 2007). Moreover, the backstepping procedure is useful for identifying parameters of nonlinear ship models (Casado and Ferreiro, 2005).

The backstepping method can provide a systematic construction process for controller design but it fails in determining the optimal values of control parameters. There seems to be no simple way to determine the control parameters. In general, adaptive fuzzy backstepping control can provide a systematic methodology of solving tracking or regulation control problems, where fuzzy systems are used to approximate unknown nonlinear functions with unknown parameters (Shaocheng et al., 2010).

The control algorithm suggested in this paper is based on the adaptive backstepping method and a genetic algorithm. The control system comprises the ship course controller. It includes the control rule and the adaptation mechanism which are derived using the backstepping method. The controller parameters were tuned using genetic algorithms (GAs) with an off-line method. The system considered fulfils basic tasks such as system stability, the tuning of controller amplifications, and system adaptation to changing environmental conditions. Thus it could hardly ensure an optimal result.

The systems presented in the literature that make use of the backstepping method were optimized by classical methods. They were usually based on the Riccati equation (Krstic and Panagiotis, 1999; Zwierzewicz, 2004), the Powell method (Casado and Ferreiro, 2005) or manual tuning procedures. The authors proposed the genetic algorithm to optimise backstepping controller parameters. This technique has not been developed with the backstepping method in marine applications. In comparison, classical methods have a major limitation especially in changing working system conditions, while the GA has a naturally built-in mechanism for adaptation.

In marine technology there is an essential problem to regard the steering gear dynamics at the design stage. This is due to its nonlinearity which is described using nonlinearity with saturation. The steering gear dynamics are not taken into consideration by the control algorithms available in the literature. Furthermore, they do not provide sufficient information on how to treat this problem. In this paper the backstepping controller takes into account, at the design stage, dynamic properties of the simplified steering gear. This enlarges the number of the recurrence. The additional step increases the computational complexity of the backstepping algorithm (a large number of uncertainties and a complex adaptation law). However, it may produce an improved system performance.

Our results are a continuation of studies initiated earlier (Witkowska et al., 2007; Witkowska and Smierzchalski, 2009). A large number of studies which discuss application of the backstepping method exist in marine systems. Although the derived control law and the adaptation law are based on the well-known backstepping method, they have not been previously considered in such a form in the literature. That is the reason for considering the Van Leeuven ship model which has the complete nonlinear maneuvering characteristics and the simplified steering gear dynamics.

\section{Mathematical ship model}

In ship motion control systems the course stabilization task is usually realized by using linear models which describe a linear relation between the rudder deflection angle and the ship course. This linear relation becomes insufficient for controlling the ship during course change manoeuvres. The instantaneous velocity of the ship decreases during such a manoeuvre. This is a result of the increasing resistance to motion which appears when the ship turns. Taking this effect into account, the synthesis of the ship course control law will be done using the nonlinear mathematical model proposed by Van Leeuven (Amerongen, 1982). This model, described by the differential equation (1), presents the nonlinear changes of the ship's angular velocity $r$ depending on instantaneous changes of the ship's velocity $U$ and the rudder deflection angle $\delta$,

$$
\begin{aligned}
T_{1}^{*} T_{2}^{*}\left(\frac{L}{U}\right)^{2} \ddot{r}+ & \left(T_{1}^{*}+T_{2}^{*}\right) \frac{L}{U} \dot{r} \\
& +\frac{U}{L} H_{N}\left(\frac{L}{U} r\right)=\frac{U}{L} K^{*} \delta \\
H_{N}\left(\frac{L}{U} r\right)= & a_{0}^{*}+a_{1}^{*} \frac{L}{U} r+a_{2}^{*}\left(\frac{L}{U}\right)^{2} r^{2} \\
& +a_{3}^{*}\left(\frac{L}{U}\right)^{3} r^{3}
\end{aligned}
$$

where $H_{N}$ is the nonlinear manoeuvring characteristic of the ship, $a_{i}^{*}$ stands for manoeuvring characteristic coefficients, $L$ is the ship length, $[\mathrm{m}], U$ is the instantaneous ship velocity, $[\mathrm{m} / \mathrm{s}], T_{1}^{*}, T_{2}^{*}, T_{3}^{*}$ are dimensionless time constants, $K^{*}$ is a dimensionless amplification.

The time constants and amplifications are determined from the results of manoeuvring tests which are performed on real objects. As regards ships, they frequently range between $0.5<T_{i}^{*}, K_{i}^{*}<2.5$. The manoeuvring characteristic coefficients $a_{i}^{*}, i \in\{0,1,2,3\}$, are usually determined in a test called the "spiral test". 
The ship actuator which controls the difference between the current and the set rudder deflection angle is the hydraulic steering gear. This device operates in the nonlinear system of the rudder deflection servomechanism and its speed is limited between $2[\mathrm{deg} / \mathrm{s}] \leq \dot{\delta}_{\max } \leq 7$ $[\mathrm{deg} / \mathrm{s}]$, with the maximum rudder deflection angle equal to $\delta_{\max }=35$ [deg]. A large number of practically used control methods is based on the assumption that the steering gear dynamics are neglected. In the present article, the mathematical model of the ship (1) is extended by including the following linear equation of steering gear dynamics (Amerongen, 1982) at the stage of designing the control law:

$$
\dot{\delta}(t)=\delta_{z}(t)-\delta(t) .
$$

The input signal for the steering gear comes from the course controller and has the form of the set rudder deflection angle $\delta_{z}$. The signal at the steering gear output is the current value of the rudder deflection angle $\delta$. The model of the ship (1) and the steering gear (3) will be used for designing the ship course control law further on in the article. Assuming that the following state variable vector is $x(t)=\left[x_{1}(t), x_{2}(t), x_{3}(t), x_{4}(t)\right]^{T}=$ $[\psi(t), r(t), \dot{r}(t), \delta(t)]^{T}$, the output signal is $y(t)=x_{1}(t)$ and the control variable is $\delta_{z}=u_{c}(t)$, we can define the model of the examined ship with the steering gear by the set of fourth-order differential equations:

$$
\begin{aligned}
\dot{x}_{1}(t) & =x_{2}(t), \\
\dot{x}_{2}(t) & =x_{3}(t), \\
\dot{x}_{3}(t) & =\left(\frac{U}{L}\right)^{3} c x_{4}(t)+\varphi^{T}(t) \theta, \\
\dot{x}_{4}(t) & =u_{c}(t)-x_{4}(t),
\end{aligned}
$$

in which, for simplifying purposes, the following substitutions were made:

$$
\begin{array}{rlrl}
a & =-\frac{T_{1}^{*}+T_{2}^{*}}{T_{1}^{*} T_{2}^{*}}, & b_{0}=-\frac{a_{0}^{*}}{T_{1}^{*} T_{2}^{*}}, & b_{1}=-\frac{a_{1}^{*}}{T_{1}^{*} T_{2}^{*}}, \\
b_{2}=-\frac{a_{2}^{*}}{T_{1}^{*} T_{2}^{*}}, & b_{3}=-\frac{a_{3}^{*}}{T_{1}^{*} T_{2}^{*}}, & c=\frac{K^{*}}{T_{1}^{*} T_{2}^{*}},
\end{array}
$$

$$
\begin{gathered}
\theta^{T}=\left[b_{0}, b_{1}, b_{2}, b_{3}, a\right] \\
\varphi^{T}=\left[\left(\frac{U}{L}\right)^{3},\left(\frac{U}{L}\right)^{2} x_{2},\left(\frac{U}{L}\right) x_{2}^{2}, x_{2}^{3},\left(\frac{U}{L}\right) x_{3}\right] .
\end{gathered}
$$

The system defines the constant ship model parameters $b_{0}, b_{1}, b_{2}, b_{3}, a$, the vector of the ship model parameters $\theta \in \mathbb{R}^{5}$ and the state vector $\varphi \in \mathbb{R}^{5}$ related to the vector of parameters $\theta$. All model nonlinearities are included in the function $\varphi$. The block diagram of the system (4)-(7) is shown in Fig. 11. The system has

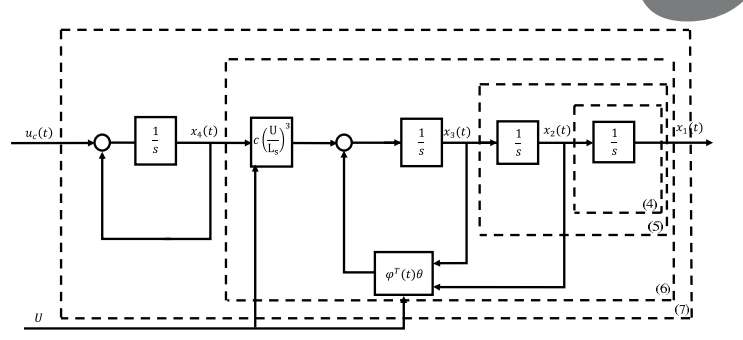

Fig. 1. Block diagram of the system (4)-(7).

a lower-triangular cascade structure. Each subsystem (4)-(7) in the presented structure can be considered a scalar and affine system with respect to the control input. Therefore the backstepping method can be successfully used for the synthesis of the control law, as the system has the required structure.

\section{Adaptive course controller}

The ship course control design process aims at determining a control law $u_{c}(t)$ with feedback. It is used for the asymptotic tracking of the set ship course $\psi_{z}(t)$ within the limits of the given tracking error. When designing the control law, we assume that we have precise information on the object, i.e., we consider the vector of parameters $\theta$ and the parameter $c$ to be known in the adopted ship model. Then the ship course control law can be derived using the classical backstepping method, as was discussed in detail by Witkowska et al. (2007), as well as Witkowska and Smierzchalski (2009).

With the reference to these works when designing in the backstepping method, we can assume that the state vector is $z(t)=\left[z_{1}(t), z_{2}(t), z_{3}(t), z_{4}(t)\right]$ with the components having the following form:

$$
\begin{aligned}
z_{1}(t) & =x_{1}(t)-\psi_{z}(t), \\
z_{2}(t) & =x_{2}(t)-\dot{\psi}_{z}(t)-\alpha_{1}(t), \\
z_{3}(t) & =x_{3}(t)-\ddot{\psi}_{z}(t)-\alpha_{2}(t), \\
z_{4}(t) & =x_{4}(t)-\left(\frac{L}{U}\right)^{3} \frac{1}{c} \psi_{z}^{(3)}(t)-\alpha_{3}(t),
\end{aligned}
$$

for which the stabilising functions are given by

$$
\begin{aligned}
& \alpha_{1}(t)=-k_{1} z_{1}(t) \\
& \alpha_{2}(t)=-k_{2} z_{2}(t)-z_{1}(t)+\dot{\alpha}_{1}(t) \\
& \alpha_{3}(t)=\left(\frac{L}{U}\right)^{3} \frac{1}{c}\left(-k_{3} z_{3}(t)-z_{2}(t)+\dot{\alpha}_{2}(t)-\varphi^{T} \theta\right)
\end{aligned}
$$

The new state variable $z_{1}$ represents the minimized ship course error, while $z_{2}$ is the stabilized angular velocity and $z_{3}$ is connected with the ship acceleration. The role of the functions $\alpha_{1}$ and $\alpha_{2}$ is to respectively stabilize the subsystems (4) and (5). The fourth 
state variable $z_{4}$ represents the deviation of the rudder deflection angle from its set value. For the variables and stabilizing functions defined as above, the derivatives of the components of the vector $z$ take the following form (Witkowska et al., 2007; Witkowska and Smierzchalski, 2009):

$$
\begin{aligned}
& \dot{z}_{1}(t)=-k_{1} z_{1}(t)+z_{2}(t) \\
& \dot{z}_{2}(t)=-k_{2} z_{2}(t)+z_{3}(t)-z_{1}(t) \\
& \dot{z}_{3}(t)=\left(\frac{U}{L}\right)^{3} c \mathrm{x}_{4}(t)+\varphi^{T}(t) \theta-\dot{\alpha}_{2}(t)-\psi_{z}^{(3)}(t) \\
& \dot{z}_{4}(t)=\dot{x_{4}}(t)-\dot{\alpha_{3}}(t)-\left(\frac{L}{U}\right)^{3} \frac{1}{c} \psi_{z}^{(4)}(t)
\end{aligned}
$$

The adaptive backstepping method is the most suitable backstepping method when precise information about the object is not known and the uncertainties have the form of unknown but constant parameters. The incomplete information about the system in the case of the ship model (4)-(7) refers to the unknown vector of parameters $\theta$ and $c$. It is assumed that the dynamics of the steering gear are known. The values of parameters can change because of the changeable operating conditions of the system caused by, e.g., a change in the ship course, the weight of the carried cargo, the water depth, or hydrodynamic conditions.

Since the first two model equations (4) and (5) do not include uncertainties, the designing procedure is identical here to that used in the classical backstepping method. We can assume that the state vector components $z_{1}, z_{2}, z_{3}$ are given by the formulae (11) and (13), while the functions $\alpha_{1}, \alpha_{2}$ are given by (15) and (16). In order to evaluate the adaptation law, we substitute the unknown parameters $\theta, c$ and $\rho=1 / c$ with their estimates $\widehat{\theta}, \widehat{c}$ and $\widehat{\rho}$. The third stabilizing function $\widehat{\alpha}_{3}$ and the fourth state variable $z_{4}$, based on the formulae (14) and (17) take the forms

$$
\begin{aligned}
\hat{\alpha}_{3}(t) & =\left(\frac{L}{U}\right)^{3} \frac{1}{\hat{c}}\left(-k_{3} z_{3}(t)-z_{2}(t)+\dot{\alpha_{2}}(t)-\varphi^{T}(t) \hat{\theta}\right), \\
z_{4}(t) & =x_{4}(t)-\left(\frac{L}{U}\right)^{3} \hat{\rho} \psi_{z}^{(3)}(t)-\hat{\alpha}_{3}(t) .
\end{aligned}
$$

Hence

$$
x_{4}(t)=\hat{\alpha}_{3}(t)+z_{4}(t)+\left(\frac{L}{U}\right)^{3} \hat{\rho} \psi_{z}^{(3)}(t) .
$$

Substituting (22) and (24) into (20), we obtain the analytical form of the derivative:

$$
\begin{aligned}
& \dot{z}_{3}(t) \\
& =\frac{c}{\hat{c}}\left(-k_{3} z_{3}(t)-z_{2}(t)-\varphi^{T}(t) \hat{\theta}+\dot{\alpha}_{2}(t)+\psi_{z}^{(3)}(t)\right) \\
& \quad+\varphi^{T}(t) \theta-\dot{\alpha}_{2}(t)+\left(\frac{U}{L}\right)^{3} c z_{4}(t)-\psi_{z}^{(3)}(t) .
\end{aligned}
$$

Let $\hat{\rho}$ be the estimate of the parameter $\rho=1 / c$. Replacing $c / \hat{c}$ by $1-c \tilde{\rho}$, where $\tilde{\rho}=\rho-\hat{\rho}$, we get

$$
\begin{gathered}
\dot{z}_{3}(t)=-k_{3} z_{3}(t)-z_{2}(t)+\left(\frac{U}{L}\right)^{3} c z_{4}(t) \\
+\varphi^{T}(t) \tilde{\theta}-c \tilde{\rho}\left(\bar{\alpha}_{3}(t)+\psi_{z}^{3}(t)\right), \\
\bar{\alpha}_{3}(t)=-k_{3} z_{3}(t)-z_{2}(t)-\varphi^{T}(t) \hat{\theta}+\dot{\alpha}_{2}(t) .
\end{gathered}
$$

The function (22) can be written in a more convenient form as

$$
\hat{\alpha}_{3}(t)=\left(\frac{L}{U}\right)^{3} \hat{\rho} \bar{\alpha}_{3}(t) .
$$

Substituting (28) into 23), the derivative $\dot{z}_{4}$ can be expressed as

$$
\begin{aligned}
\dot{z}_{4}(t)= & \dot{x}_{4}(t)-\left(\frac{L}{U}\right)^{3} \hat{\rho}\left(\dot{\bar{\alpha}}_{3}(t)+\psi_{z}^{(4)}(t)\right) \\
& -\left(\frac{L}{U}\right)^{3} \hat{\hat{\rho}}\left(\bar{\alpha}_{3}(t)+\psi_{z}^{(3)}(t)\right) \\
\dot{\bar{\alpha}}_{3}(t)= & \frac{\partial \bar{\alpha}_{3}}{\partial x_{1}} x_{2}(t)+\frac{\partial \bar{\alpha}_{3}}{\partial x_{2}} x_{3}(t) \\
& +\frac{\partial \bar{\alpha}_{3}}{\partial x_{3}}\left(\left(\frac{U}{L}\right)^{3} c x_{4}(t)+\varphi^{T}(t) \theta\right) \\
& +\frac{\partial \bar{\alpha}_{3}}{\partial \hat{\hat{\theta}}}+\frac{\partial \bar{\alpha}_{3}}{\partial \psi_{z}} \dot{\psi}_{z}+\frac{\partial \bar{\alpha}_{3}}{\partial \dot{\psi}_{z}} \ddot{\psi}_{z}+\frac{\partial \bar{\alpha}_{3}}{\partial \ddot{\psi}_{z}} \psi_{z}^{(3)}
\end{aligned}
$$

From Eqn. (7), we get

$$
\begin{aligned}
\dot{z}_{4}(t)= & u_{c}(t)-x_{4}(t)-\left(\frac{L}{U}\right)^{3} \hat{\rho}\left(\dot{\bar{\alpha}}_{3}(t)+\psi_{z}^{(4)}(t)\right) \\
& -\left(\frac{L}{U}\right)^{3} \dot{\hat{\rho}}\left(\bar{\alpha}_{3}(t)+\psi_{z}^{(3)}(t)\right) .
\end{aligned}
$$

Then, assuming the control law $u_{c}$ in the form

$$
\begin{aligned}
u_{c}= & -k_{4} z_{4}(t)+x_{4}(t)-\left(\frac{U}{L}\right)^{3} \hat{c}_{3}(t) \\
& +\left(\frac{L}{U}\right)^{3} \hat{\rho}\left(\frac{\partial \bar{\alpha}_{3}}{\partial x_{1}} x_{2}(t)+\frac{\partial \bar{\alpha}_{3}}{\partial x_{2}} x_{3}(t)\right. \\
& +\frac{\partial \bar{\alpha}_{3}}{\partial x_{3}}\left(\left(\frac{U}{L}\right)^{3} \hat{c} x_{4}(t)+\varphi^{T}(t) \hat{\theta}\right) \\
& +\frac{\partial \bar{\alpha}_{3}}{\partial \hat{\theta}} \dot{\hat{\theta}}+\frac{\partial \bar{\alpha}_{3}}{\partial \psi_{z}} \dot{\psi}_{z}+\frac{\partial \bar{\alpha}_{3}}{\partial \dot{\psi}_{z}} \ddot{\psi}_{z}+\frac{\partial \bar{\alpha}_{3}}{\partial \ddot{\psi}_{z}} \psi_{z}^{3} \\
& \left.+\psi_{z}^{(4)}(t)\right)+\left(\frac{L}{U}\right)^{3} \dot{\hat{\rho}}\left(\bar{\alpha}_{3}(t)+\psi_{z}(t)\right)
\end{aligned}
$$

and substituting this into 31, we get the formula for the derivative of the component $z_{4}(t)$,

$$
\begin{aligned}
\dot{z}_{4}(t)= & -k_{4} z_{4}(t)-\left(\frac{U}{L}\right)^{3} c z_{3}(t)+\tilde{c}\left(\left(\frac{U}{L}\right)^{3} z_{3}(t)\right. \\
& \left.-\hat{\rho} \frac{\partial \bar{\alpha}_{3}}{\partial x_{3}} x_{4}(t)\right)-\left(\frac{L}{U}\right)^{3} \hat{\rho} \frac{\partial \bar{\alpha}_{3}}{\partial x_{3}} \varphi^{T}(t) \tilde{\theta}
\end{aligned}
$$


The Lapunov function for the entire system, complemented by the squares of the unknown parameter errors, takes the form

$$
\begin{aligned}
V_{a}(t)= & \frac{1}{2} z_{1}^{2}(t)+\frac{1}{2} z_{2}^{2}(t)+\frac{1}{2} z_{3}^{2}(t) \\
& +\frac{1}{2} z_{4}^{2}(t)+\frac{1}{2} \tilde{\theta}^{T} \Gamma^{-1} \tilde{\theta}+\frac{|c|}{2 \gamma_{\mathrm{c}}} \tilde{\rho}^{2}+\frac{1}{2 \gamma_{\mathrm{c}}} \tilde{c}^{2}
\end{aligned}
$$

where $\Gamma=\operatorname{diag}\left(\gamma_{0}, \gamma_{1}, \gamma_{2}, \gamma_{3}, \gamma_{a}\right)>0$ is a diagonal matrix with positively defined coefficients and $\gamma_{c}>0$.

Its derivative is given by the formula

$$
\begin{aligned}
& \dot{V}_{a}(t) \\
& =z_{1}(t) \dot{z}_{1}(t)+z_{2}(t) \dot{z_{2}}(t)+z_{3}(t) \dot{z_{3}}(t) \\
& \quad+z_{4}(t) \dot{z_{4}}(t)+\tilde{\theta}^{T} \Gamma^{-1} \dot{\tilde{\theta}}+\frac{|c|}{\gamma_{c}} \tilde{\rho} \dot{\tilde{\rho}}+\frac{1}{\gamma_{c}} \tilde{c} \dot{\tilde{c}} .
\end{aligned}
$$

Since the parameters $\theta, c$ and $\rho$ are constant or change slowly, we can approximately assume that $\dot{\tilde{\theta}}=-\dot{\hat{\theta}}, \dot{\tilde{\rho}}=$ $-\dot{\hat{\rho}}, \dot{\tilde{c}}=-\dot{\hat{c}}$. Hence

$$
\begin{aligned}
& \dot{V}_{a}(t) \\
& =z_{1}(t) \dot{z_{1}}(t)+z_{2}(t) \dot{z_{2}}(t)+z_{3}(t) \dot{z_{3}}(t) \\
& \quad+z_{4}(t) \dot{z}_{4}(t)-\tilde{\theta}^{T} \Gamma^{-1} \dot{\hat{\theta}}-\frac{|c|}{\gamma_{c}} \tilde{\rho} \dot{\hat{\rho}}-\frac{1}{\gamma_{c}} \tilde{c} \dot{\hat{c}} .
\end{aligned}
$$

Replacing the derivatives $\dot{z}_{i}, i \in\{1,2,3,4\}$ by the formulae (18), (19), (26) and (33), we arrive at

$$
\begin{aligned}
\dot{V}_{a}(t) & \\
= & -\sum_{i=1}^{4} k_{i} z_{i}^{2}(t)+\tilde{\theta}^{T}\left(\varphi(t) z_{3}(t)\right. \\
& \left.-\left(\frac{L}{U}\right)^{3} \hat{\rho} \frac{\partial \bar{\alpha}_{3}}{\partial x_{3}} \varphi(t) z_{4}(t)-\Gamma^{-1} \dot{\hat{\theta}}\right) \\
& -c \tilde{\rho}\left(\left(\bar{\alpha}_{3}+\psi_{z}^{(3)}\right) z_{3}(t)+\frac{|c|}{c \gamma_{c}} \dot{\hat{\rho}}\right) \\
& +\tilde{c}\left(\left(\frac{U}{L}\right)^{3} z_{3}(t) z_{4}(t)-\hat{\rho} \frac{\partial \bar{\alpha}_{3}}{\partial x_{3}} x_{4}(t) z_{4}(t)-\frac{1}{\gamma_{c}} \dot{\hat{c}}\right) .
\end{aligned}
$$

To eliminate the expressions containing the estimation errors $\tilde{\theta}, \tilde{c}$ and $\tilde{\rho}$ in the formula (37), we have the following adaptation laws:

- for vector parameter $\theta$ :

$$
\dot{\hat{\theta}}=\Gamma \varphi(t)\left(z_{3}(t)-\left(\frac{L}{U}\right)^{3} \hat{\rho} \frac{\partial \bar{\alpha}_{3}}{\partial x_{3}} z_{4}(t)\right),
$$

- for parameter $\rho$ :

$$
\dot{\hat{\rho}}=-\gamma_{c} \operatorname{sgn}(c)\left(\bar{\alpha}_{3}+\psi_{z}^{(3)}\right) z_{3}(t),
$$

- for parameter $c$ :

$$
\dot{\hat{c}}=\gamma_{c}\left(\left(\frac{U}{L}\right)^{3} z_{3}(t)-\hat{\rho} \frac{\partial \bar{\alpha}_{3}}{\partial x_{3}} x_{4}(t)\right) z_{4}(t) .
$$

In the simulation analyses, the values of the estimates $\theta, c$ and $\rho$ were calculated from Eqns. (37)- (40). These approximate values were directly used in the control law (32). The values of the estimates strongly depend on the coefficients of the matrix $\Gamma$. The characteristic of the estimated parameters in the adaptive system is remarkably affected by the changeable amplification coefficients in the adaptation loop. When these coefficients are too large, over-regulation can take place and the quality of control can become worse. A series of experimental tests were performed to determine the values for the coefficients in the matrix $\Gamma$. The obtained laws guarantee that the derivative of the Lyapunov function is nonpositive and has the following final form:

$$
\dot{V}_{a}(t)=-\sum_{i=1}^{4} k_{i} z_{i}^{2}(t) \leq 0 .
$$

If the values of the estimates are determined correctly, the estimation errors $\tilde{\theta}=\theta-\hat{\theta}, \tilde{\rho}=\rho-\hat{\rho}, \tilde{c}=$ $c-\hat{c}$ are zero and $\theta=\hat{\theta}, \rho=\hat{\rho}, c=\hat{c}$. In consequence, the control law $u_{c}$ guarantees the convergence of the output signal $x_{1}$ to the set value $\psi_{z}(t)$ as $t \rightarrow \infty$. The state equations have the form of the nonlinear matrix equation, in which the main matrix is the sum of a diagonal matrix with negative definite elements and a skew-symmetric matrix:

$$
\begin{aligned}
& {\left[\begin{array}{c}
\dot{z}_{1} \\
\dot{z}_{2} \\
\dot{z}_{3} \\
\dot{z}_{4}
\end{array}\right]} \\
& =\left[\begin{array}{cccc}
-k_{1} & 1 & 0 & 0 \\
-1 & -k_{2} & 1 & 0 \\
0 & -1 & -k_{3} & \left(\frac{U}{L}\right)^{3} c \\
0 & 0 & -\left(\frac{U}{L}\right)^{3} c & -k_{4}
\end{array}\right]\left[\begin{array}{c}
z_{1} \\
z_{2} \\
z_{3} \\
z_{4}
\end{array}\right] \\
& +\left[\begin{array}{c}
0 \\
0 \\
1 \\
-\left(\frac{L}{U}\right)^{3} \hat{\rho} \frac{\partial \bar{\alpha}_{3}}{\partial x_{3}}
\end{array}\right] \varphi^{T} \tilde{\theta}-c\left[\begin{array}{c}
0 \\
0 \\
\bar{\alpha}_{3}+\psi_{z}^{(3)} \\
0
\end{array}\right] \tilde{\rho} \\
& +\left[\begin{array}{c}
0 \\
0 \\
0 \\
\left(\frac{U}{L}\right)^{3} z_{3}(t)-\hat{\rho} \frac{\partial \bar{\alpha}_{3}}{\partial x_{3}} x_{4}(t)
\end{array}\right] \tilde{c},
\end{aligned}
$$

where $k_{1}, k_{2}, k_{3}, k_{4}>0$ are design parameters. 


\section{Control system structure}

The correctness and quality of the designed adaptive ship course controller were checked by using a simplified (1) and full-scale mathematical model as a control system. A general diagram of the ship course control system is shown in Fig. 2] The system includes the mathematical model of ship dynamics, complemented by the model of environmental disturbances and the model of the steering gear. The control system comprises the ship course controller which includes the control rule derived using the backstepping method with the control law (32).

The model parameters were estimated using the adaptive backstepping method. The adaptation mechanism of ship model parameters is determined by the adaptation rule, according to the formulae (38)-(40). The controller amplifications were selected using genetic algorithms with an off-line method. The obtained amplifications were used as the input data for the backstepping controller. The model of environmental disturbances included wind and wave disturbances which were the result of wind. A third-order low passing filter of frequency $0.05[\mathrm{rad} / \mathrm{s}]$ was defined in the system to facilitate obtaining smooth characteristics of the reference signal of the course and its derivatives.

When presenting the simulation tests we shall discuss in detail the following issues: the initial values of the estimated parameters, the matrix of adaptation coefficients, and the set parameters. The measured signals were filtered before passing to the feedback loop to reduce excessive movements of the rudder blade. For this purpose, we used a low-pass filter which enables obtaining a slight shift of the phase and amplitude of the measured and filtered angular velocity with reference to the control signal at the input to the steering gear. The system was implemented in the Matlab/Simulink and $\mathrm{C}++$ packages.

4.1. Full-scale mathematical model. The full-scale mathematical model is a container ship model bearing the shipyard symbol B-481. It includes the hull dynamics and the main propulsion system which consists of a single adjustable blade propeller, rudder and two lateral thrusters: on the bow and stern sides. The effect of disturbances of the environmental origin (wind, waves, sea current) and changes in dynamics caused by shallow water were also taken into account. The model allows analysing the behaviour of the ship for two load conditions: ballast and full load. The equations composing the mathematical model of the B-481 vessel are presented by Galbas (1988). Basic design parameters of the real ship are given in Table 1 .

4.2. Steering gear dynamic. Both simplified and full-scale models were complemented by the dynamics of the steering gear, described by Velagic et al. (2003)
Table 1. Basic design parameters of the B-481 type ship.

\begin{tabular}{|c||c|c|c|c|}
\hline & & & Ballast & Full load \\
\hline Overall length & $L$ & {$[\mathrm{~m}]$} & 181 & 181 \\
\hline $\begin{array}{c}\text { Length between } \\
\text { perpendiculars }\end{array}$ & $L_{p}$ & {$[\mathrm{~m}]$} & 165 & 165 \\
\hline Length on waterline & $L_{w}$ & {$[\mathrm{~m}]$} & 161.6 & 173.15 \\
\hline Width & $B$ & {$[\mathrm{~m}]$} & 28.2 & 28.2 \\
\hline Bow draught & $T_{D}$ & {$[\mathrm{~m}]$} & 5.55 & 9.64 \\
\hline Stern draught & $T_{R}$ & {$[\mathrm{~m}]$} & 6.7 & 9.64 \\
\hline Displacement & $W$ & {$\left[\mathrm{~m}^{3}\right]$} & 16400 & 28350 \\
\hline $\begin{array}{c}\text { Distance of centre } \\
\text { of gravity from } \\
\text { midship section plane }\end{array}$ & $x_{G}$ & {$[\mathrm{~m}]$} & 4.6 & 5.6 \\
\hline
\end{tabular}

and schematically shown in Fig. 3. The simulation model of the steering gear consisted of two electro-hydraulic arrangements: a telemotor position servo and a rudder servo actuator. The value of parameters for the obtained steering machine were the following: $K=4$ [deg], $D B=1[\mathrm{deg}], H=0.8[\mathrm{deg}], P B=1.43[\mathrm{deg} / \mathrm{s}]$, $N=0.8[\mathrm{deg}], \delta_{\max }=20[\mathrm{deg}]$.

\section{Optimization process}

The classical genetic algorithm was applied for tuning backstepping controller parameters $k_{1}, k_{2}, k_{3}, k_{4}$ with an off-line method. Genetic operations comprised classical selection, crossover and mutation. For more information about genetic operations, see the work of Witkowska et al. (2007).

5.1. Cost function. In the genetic method all individuals (here sets of parameters $k_{1}, k_{2}, k_{3}, k_{4}$ ) are evaluated by the cost function which should reflect optimization criteria. The basic criterion is the economic one. The autopilots aim to ensure such control of the way which enables the least deviation from the setpoint by decreasing the activity of the rudder. This manner of control prevents excessive losses of speed, and reduces the travel time and fuel consumption. The cost of the function is computed according to the following formula (Amerongen, 1982):

$$
J_{c_{1}}=\frac{0.0076}{T} \int_{0}^{T}\left(\Delta \psi^{2}+\lambda \delta^{2}\right) \mathrm{d} \tau,
$$

where $\lambda$ is the scale factor, $\lambda=0.1, \Delta \psi$ is the course error, $\delta$ is the rudder deflection angle, $J_{c_{1}}$ is the loss of speed [\%].

Van Amerongen and Van Nauta Lemke (1978) proposed another evaluation function described by the formula

$$
J_{c_{2}}=\frac{0.0076}{T} \int_{0}^{T}\left(\Delta \psi^{2}+\lambda_{1} \Delta \tilde{\dot{\psi}}^{2}+\lambda_{2} \delta^{2}\right) \mathrm{d} \tau,
$$




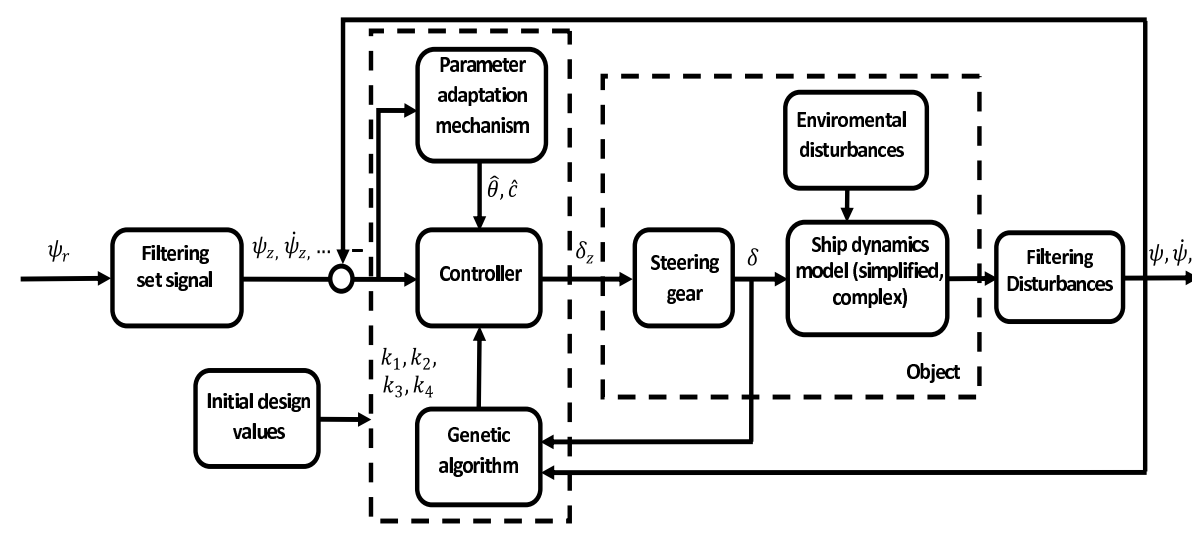

Fig. 2. General scheme of the ship course control system.

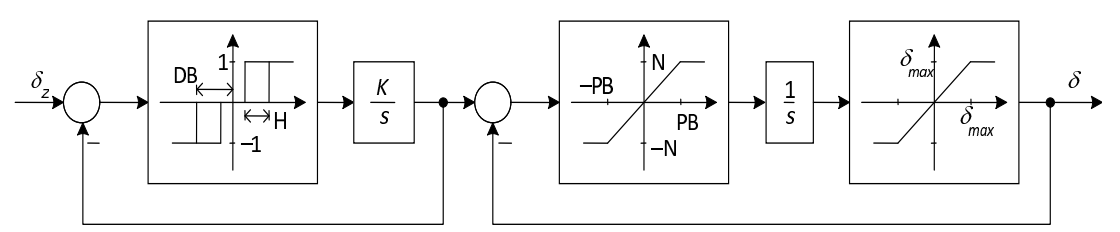

Fig. 3. Block diagram of the steering gear.

where $\tilde{\dot{\psi}}$ is the wave filtered signal of rate of turn.

The loss of speed may be also expressed as a function of the turn rate caused by rudder motion. The component which is caused by disturbances, mainly the waves, should be removed. It is appropriate to reflect the wave filtered signal of the rate of turn in the evaluation function. Based on the available literature (Amerongen, 1982), the coefficients weight, for container vessels with a length of about $200[\mathrm{~m}]$, can be assumed as $\lambda_{1}=1.6, \lambda_{2}=6$.

5.2. Optimization test. Several manoeuvre tests using the GA were made in the system shown in Fig. 2, On the basis of the preliminary experiments with different values of the initial GA parameters, it was assumed that the probability of crossover was $p_{c c}=0.40$, the probability of mutation $p_{m}=0.01$, the initial population size pop $_{\text {size }}=$ 200 and the space of solutions $\Omega=(1,50) \times(1,50) \times$ $(1,50) \times(0,5)$. The maximum number of generations for each test was equal to 100 . If, during the learning process, the value of the quality coefficient $J_{c 1}$ was on the same level up to the first digit place within minimum 5 tests, the process was stopped.

The tests were performed for various loading conditions of the vessel, various sea states and different values of the specified course of the ship. In each case it was assumed that the desired propeller revolution was $n_{z}^{s}=1.006[\mathrm{obr} / \mathrm{s}]$. The configuration of the GA was switched 15 times for each manoeuvre trial. First, the system shown in Fig. 2] was tested in sea condition $5\left[^{\circ} B\right]$ and wind direction 0 [deg].

The results are shown in Figs. 4 and 5. The ship dynamic characteristic equations comprise parameters estimated for ballasting state. The ship course rapidly changed by 10 [deg]. The adjusted control parameters $k_{1}, k_{2}, k_{3}, k_{4}$ are given in Table 2 for the full-scale and the simplified model as an object. These are the parameter values at which the minimum values of the quality coefficient $J_{c 1}$ were obtained at the stage of tuning with the aid of the genetic algorithm. The time-histories of system responses to a given rudder deflection angle were recorded during the test within the time interval of 500 [s] with the sampling period 0.5 [s]. They are shown in Figs. 4 and 5 The system operation was compared with the simplified and the full-scale model.

The numerically obtained results determine approximate characteristics for the two models without overregulation. Tracking the set course values was correct. The maximum course error did not exceeded 1 [deg]. With reference to the steering gear operation, the actuator worked without chattering and the rudder deflection angle change was a smooth function. The overall performance was satisfactory. There is no actuator saturation observed up to the maximum value of the rudder deflection angle 20 [deg]. In order to evaluate the intermediate intervals in the ship course time-characteristics, the rapid changes of the set course were simulated using direct control quality coefficients: the build-up time $t_{n}$, the value of the first overregulation $M_{p}$ with the accuracy of 0.01 , and the control time $t_{R}$ determined for $1 \%$ of the intermediate time-history part after changing the set course.

Regulations on Classification and Building of SeaGoing Vessels assume that the set course is kept with the 

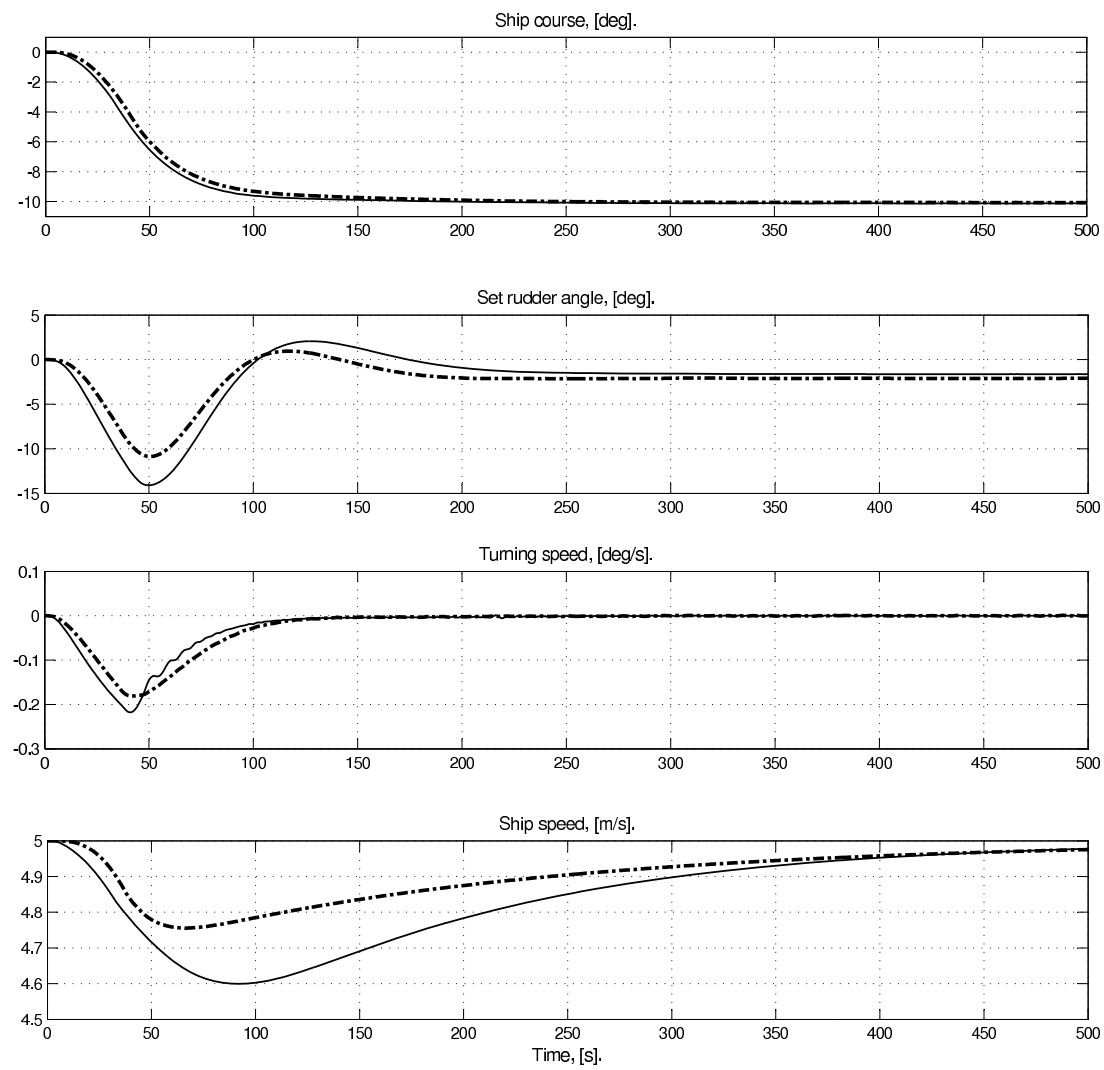

Fig. 4. Time-histories of the ship course, rudder deflection angle, turning speed and ship speed during a rapid change of the set course: full-scale model (solid line), simplified model (dashed line).

Table 2. Adjustable parameters and the cost function value (in sea conditions $5\left[{ }^{0} \mathrm{~B}\right]$ and wind direction 0 [deg]).

\begin{tabular}{|l||c|c|c|c|c|c|c|}
\hline & $k_{1}$ & $k_{2}$ & $k_{3}$ & $k_{4}$ & $J_{c 1}[\%]$ & $J_{c 2}[\%]$ & $N_{\max }$ \\
\hline \hline Full-scale model & 25.4839 & 23.2258 & 21 & 0.7 & $12.556 .10-3$ & 0.75333 & 34 \\
\hline Simplified model & 29.0645 & 24.3871 & 35 & 1.204 & $9.3995 .10-3$ & 0.56395 & 29 \\
\hline
\end{tabular}

Table 3. Values of temporal quality coefficients.

\begin{tabular}{|l||c|c|c|}
\hline & $t_{n}[\mathrm{~s}]$ & $M_{p}[\%]$ & $t_{R}[\mathrm{~s}]$ \\
\hline \hline Full-scale model & 64 & - & 198 \\
\hline Simplified model & 59 & - & 298 \\
\hline
\end{tabular}

maximum single amplitude of $1.5[\mathrm{deg}]$ and its accuracy is within $1[\mathrm{deg}]$. The values of the temporal quality coefficients for the test are given in Table 3. The speed of the ship decreased during the course change manoeuvre. After completing the manoeuvre the speed returned to the initial value observed before the manoeuvre (after about $300[\mathrm{~s}])$. The differences in the obtained time-histories of the instantaneous model ship speed resulted in part from the adopted simplified assumptions about the ship model. These can be observed in Fig. 4

The state vector components $z=\left[z_{1}, z_{2}, z_{3}, z_{4}\right]$ tend to zero. This suggests that the system behaves in a stable way. The initial values of the model parameters, for which the controller settings were selected in the control structure shown in Fig. $2 b_{0}(0)=$ $17.2103, b_{1}(0)=-5.4348, b_{2}(0)=0.017935, b_{3}(0)=$ $-0.0086957, a(0)=-12.6087, c(0)=8.6957, \rho(0)=$ 0.1150 . The assumed initial values of the estimated parameters were equal to $25 \%$ of the real parameter value. Both real and accurate values of the estimated parameters, which were obtained in the tests for the full-scale model and the simplified model are given in Tables 4 and $5 . \quad$ The backstepping method does not guarantee the convergence of all parameters to their actual values. In the examined case, the convergence of the estimated parameters $b_{1}, b_{2}, b_{3}, a, c$ to the real values after about 60 [s] are secured by the correct selection of the adaptation matrix. The value of the parameter $\rho$ changes 
Table 4. Real and estimated values of the full-scale model parameters obtained for the assumed amplifications.

\begin{tabular}{|l||c|c|c|c|c|c|c|}
\hline Parameters & $b_{0}[\mathrm{deg}]$ & $b_{1}[-]$ & $b_{2}[1 / \mathrm{deg}]$ & $b_{3}\left[1 / \mathrm{deg}^{2}\right]$ & $a[-]$ & $c[-]$ & $\rho[-]$ \\
\hline \hline Real & 68.8413 & -21.7391 & 0.0717 & -0.0347 & -50.4348 & 34.7826 & 0.02875 \\
\hline Estimated & 68.8452 & -21.738 & 0.07571 & -0.03449 & -50.4386 & 34.1723 & 0.1397 \\
\hline & $\gamma_{0}$ & $\gamma_{1}$ & $\gamma_{2}$ & $\gamma_{3}$ & $\gamma_{a}$ & $\gamma_{c}$ & $\gamma_{c}$ \\
\hline Amplification & $6.184 \cdot 10^{-11}$ & $63.55 \cdot 10^{-10}$ & $1 \cdot 10^{-8}$ & $8 \cdot 10^{-7}$ & $62.63 \cdot 10^{-9}$ & $58.12 \cdot 10^{-10}$ & $58.12 \cdot 10^{-10}$ \\
\hline
\end{tabular}

Table 5. Real and estimated values of the simplified model parameters obtained for the assumed amplifications.

\begin{tabular}{|l||c|c|c|c|c|c|c|}
\hline Parameters & $b_{0}[\mathrm{deg}]$ & $b_{1}[-]$ & $b_{2}[1 / \mathrm{deg}]$ & $b_{3}\left[1 / \mathrm{deg}^{2}\right]$ & $a[-]$ & $c[-]$ & $\rho[-]$ \\
\hline \hline Real & 68.8413 & -21.7391 & 0.0717 & -0.0347 & -50.4348 & 34.7826 & 0.02875 \\
\hline Estimated & 68.8433 & -21.7399 & 0.07188 & -0.03466 & -50.4348 & 34.7827 & 0.1284 \\
\hline & $\gamma_{0}$ & $\gamma_{1}$ & $\gamma_{2}$ & $\gamma_{3}$ & $\gamma_{a}$ & $\gamma_{c}$ & $\gamma_{c}$ \\
\hline Amplification & $4.414 \cdot 10^{-11}$ & $8.64 \cdot 10^{-9}$ & $1.57 \cdot 10^{-8}$ & $3.86 \cdot 10^{-7}$ & $2.893 \cdot 10^{-8}$ & $9.483 \cdot 10^{-9}$ & $9.483 \cdot 10^{-9}$ \\
\hline
\end{tabular}
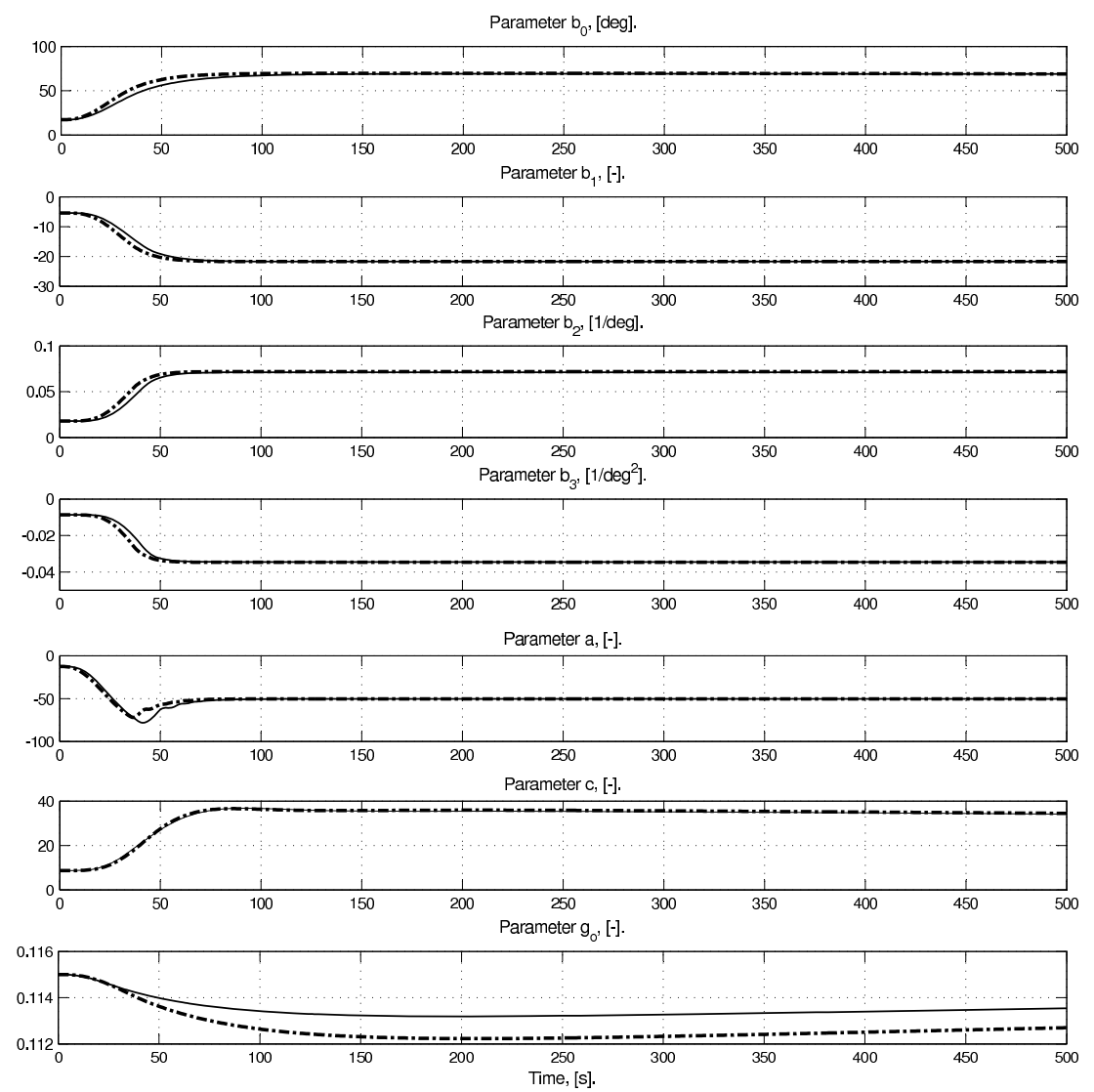

Fig. 5. Time-histories of estimated parameters at a rapid change of the set course: full-scale model (solid line), simplified model (dashed line).

relatively slowly and does not reach the value $1 / c$. The time-histories of changes in the estimated parameters for the simplified model and the full-scale model are shown in Fig. 5 .

\section{Simulation tests}

The simulation tests aim at checking the operation correctness of the backstepping controller with the estimating mechanism of model ship parameters. The issues examined in the tests include the effect of identifying accuracy of model parameters on the control system dynamics, the convergence to real parameter values and the ability to follow the fast changes in the set course. The following assumptions were made in the simulation tests:

- The dynamic model and parameters of the water system in the vicinity of the ship are not precisely known (there are only known the approximate values 
of the real model ships parameters. They have the $8 \%$ deflection in certain disturbances).

- All state variables are measurable. There is a measuring system which provides accurate information about the current ship position, course, and linear and angular speed.

These assumptions were considered in the manoeuvring test. The next test was performed for the programmed inertial change of the set course. The disturbances were corresponding to $7\left[{ }^{\circ} \mathrm{B}\right]$ and the wind direction to $0[\mathrm{deg}]$. The time-histories shown in Figs. 6 and 7 confirm the good ability of the control system to follow fast changes of the set course (by 250 [s]). The application of adaptive controllers enables keeping the optimal course independently of object parameters changes. When the conditions of object operation change, for instance, due to the ship course change or intensification of disturbances, the values of the parameters change in a limited manner (Fig. 6). The estimated parameters change slowly within time intervals of steady-load operation of the object. The presence of a small drift was recorded for the parameter $b_{0}$.

A method which is used on ships to minimise the drift phenomenon is stopping the adaptation process for some time after the manoeuvre has been completed. The undesired phenomena which was the consequence of the imperfection of the model can be minimised using the so-called robust modifications of estimation algorithms, such as sigma modification, projection of parameters, epsilon modification, dead zone modification. They are not analysed in the present article. During ship steering, the wind and waves considerably affect the rudder deflection angle. The controller characteristics can be improved, e.g., by extending the steering gear dead zone. This way some astatism of the control system can be reached with respect to disturbances.

\section{Conclusions}

The article presents the backstepping method based on ship course control system and the method providing a automatic selection of backstepping controller parameters to adapt to changing conditions of system operation. All that is done with the aid of adaptive methods and genetic algorithms. The backstepping method reduces the system to the form of the linear system described using new state variables. The obtained control law is nonlinear. The presented system reveals high sensitivity to control parameter changes. The backstepping method enables designing a stable control system which follows changes in system dynamics resulting from environmental disturbances.

In the presented control system, the genetic algorithm enables selecting controller settings, while the adaptation law enables determining model parameters. The ship course control algorithm, worked out using the adaptive backstepping method, ensures correct control system operation and the tracking of the set course value. The system automatically adjusts parameters of the controller to changing characteristics of the controlled object and the environment. When analysing the problem of resistance, the nonlinearities existing in the system are used during the designing process of the control law with the aid of the backstepping method. This is of high importance when examining control systems' resistance to modelling errors. Moreover, it is not necessary to have precise information on forces and moments affecting the ship. The presented results reveal that the system adapts well to changing environmental conditions. Furthermore, the obtained results can be fully accepted in real navigational practice.

The authors proposed the genetic algorithm to tune the controller gains. This technique has not been developed in marine applications for the above-mentioned purpose by using the backstepping method. This has advantages and disadvantages and is debatable. The genetic algorithm applied is very general. It does not impose special requirements regarding the goal function. With the aim of unconstrained optimization and low-dimensional problems, it may be one of the most efficient algorithms for nonlinear global optimization tasks. Of course, this depends on an efficient implementation.

The task of optimizing the parameters of the proposed backstepping controller is a precise example of those characteristics. This is because of the low dimensionality, non-linearity and complexity. Classical methods have a major limitation, especially in changing system working conditions, while the GA has a naturally built-in mechanism of adaptation to changing working conditions. The genetic algorithm is rather slow and usually requires a relatively long computational time. However, the application of a genetic algorithm in real time will succeed only with a proper technical implementation of the autopilot system. This is a subject of future studies.

\section{References}

Alfarocid, E., McGookin, E.W., Murray-Smith, D.J. and Fossen, T.I. (2005). Genetic algorithms optimisation of decoupled sliding model controllers simulated and real results, Control Engineering Practice 13(6): 739-748.

Almeida, J., Silvestre, C. and Pascoal, A. (2007). Path-following control of fully-actuated surface vessels in the presence of ocean currents, Proceedings of the IFAC Conference on Control Applications in Marine Systems, CAMS, Bol, Croatia, (on CD-ROM).

Amerongen, J. (1982). Adaptive Steering of Ships-A Model Reference Approach to Improved Maneuvering and Eco- 

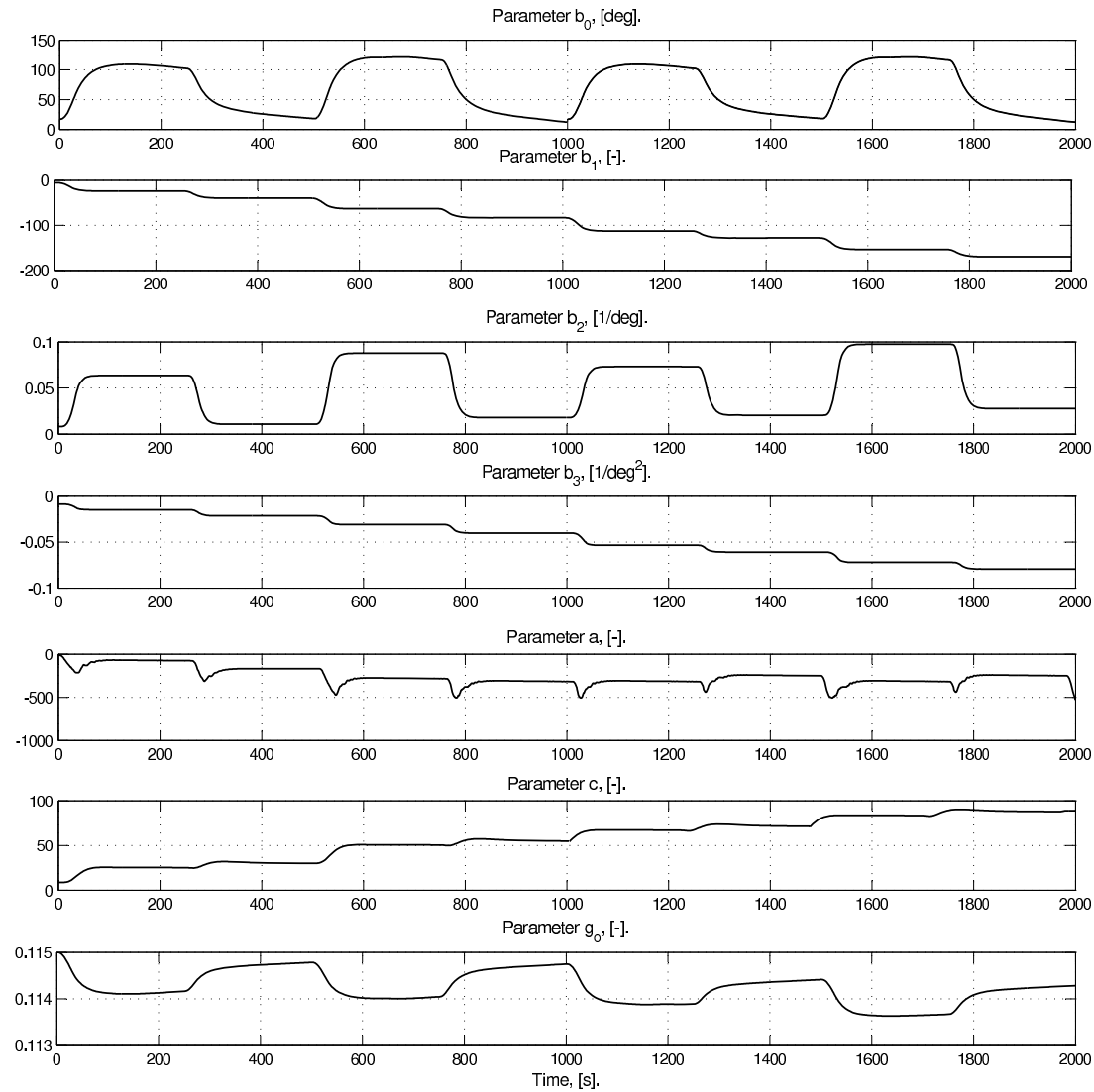

Fig. 6. Time-histories of the estimated parameters during a programmed change of the set course for the full-scale model.
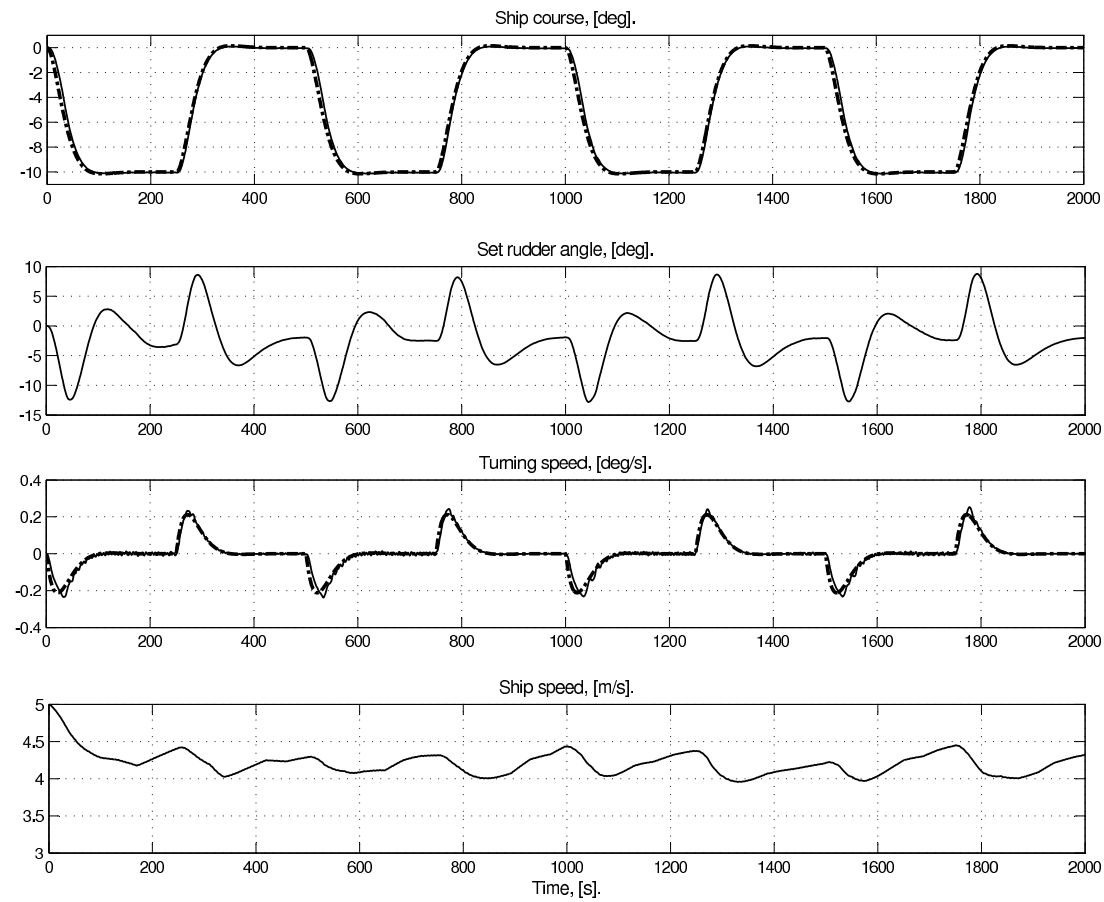

Fig. 7. Time-histories of the ship course, rudder deflection angle, turning speed, ship speed during a programmed change of the set ship course. 
nomical Course Keeping, Ph.D. thesis, Delft University of Technology, Delft.

Bibuli, M., Caccia, M. and Lapierre, L. (2007). Path-following control of fully-actuated surface vessels in the presence of ocean currents, Proceedings of the IFAC Conference on Control Applications in Marine Systems, Bol, Croatia, (on CD-ROM).

Casado, M.H. and Ferreiro, R. (2005). Identification of the nonlinear ship model parameters based on the turning test trials and the backstepping procedure, Elsevier Ocean Engineering 32(11-12): 1350-1369.

Chen, W., Zhou, F., Li, Y. and Song, R. (2008). The ship nonlinear course system control based on auto disturbance rejection controller, Proceedings of the 7th World Congress on Intelligent Control and Automation, Chongqing, China, pp. 6454-6458.

Do, K.D., Jiang, Z. and Pan, J. (2004). Robust adaptive path following of underactuated ships, Automatica 40(6): 929-944.

Du, J.L., Guo, C.Y. and Zhao, S. (2004). Adaptive robust nonlinear design of course keeping ship steering autopilot, 8th Control Automation Robotics and Vision Conference, ICARCV, Kunming, China, Vol. 1, pp. 13-18.

Fossen, T.I. and Strand, J.P. (1999). A tutorial on nonlinear backstepping: Applications to ship control, Modelling, Identification and Control 20(2): 83-135.

Galbas, J. (1988). Synthesis of Precise Ship Control Using Thrusters, Ph.D. thesis, Gdańsk University of Technology, Gdańsk, (in Polish).

Grimble, M., Zhang, Y. and Katebi, M.R. (1993). $H \infty$-based ship autopilot design, Ship Control Symposium, Ottawa, Canada, pp. 678-683.

Han, J.Q. (2002). From PID technique to active disturbance rejection control technique, Control Engineering of China 9(3): 13-18.

Han, Y., Xiao, H., Wang, C. and Zhou, F. (2009). Design and simulation of ship course controller based on auto disturbance rejection control technique, Proceedings of the IEEE International Conference on Automation and Logistics, Shenyang, China, pp. 686-691.

Karr, C.L. (1991). Design of an adaptive fuzzy logic controller using a genetic algorithm, International Conference on Genetic Algorithms, ICGA, San Diego, CA, USA, pp. 450-457.

Kokotovic, P. and Arcak, M. (2001). Constructive nonlinear control: A historical perspective, Automatica 37(5): 637-662.

Krstic, M., Kanellakopulos, I. and Kokotovic, P.V. (1995). Nonlinear and Adaptive Control Design, John Wiley and Sons Ltd., New York, NY.

Krstic, M. and Panagiotis, T. (1999). Inverse optimal stabilization of a rigid spacecraft, IEEE Transactions on Automatic Control 44(5): 1042-1043.

McGookin, E., Murray-Smith, D., Li, Y. and Fossen, T.I. (2000). Ship steering control system optimisation using genetic algorithms, Control Engineering Practice 8(4): 429-443.
Messer, A. and Grimble, M. (1993). Introduction to robust ship track-keeping control design, Transactions of the Institute of Measurement and Control 15(3): 104-110.

Pettersen, K. and Nijmeijer, H. (2004). Introduction to robust ship track-keeping control design, Transactions of the Institute of Measurement and Control 20(4): 189-199.

Richter, R. and Burns, R. (1993). An artificial neural network autopilot for small vessels, Proceedings of the 1st Conference of the UK Simulation Society, Edinburgh, UK, pp. 168-172.

Ruan, J.H. (2006). The design of ship course intelligent controller based on FNN of non-linear system, Journal of Shandong Jiaotong University 14(4): 29-33.

Shaocheng, T., Changliang, L. and Yongming, L. (2010). Robust adaptive fuzzy filters output feedback control of strict feedback nonlinear systems, International Journal of Applied Mathematics and Computer Science 20(4): 637-653, DOI: $10.2478 / \mathrm{v} 10006-010-0047-\mathrm{x}$.

Simensen, R. (1995). Simulation of artificial neural networks for ship steering control, Proceedings of the 2nd Conference of the UK Simulation Society, Edinburgh, UK, pp. 65-72.

Tomera, M. (2010). Nonlinear controller design of a ship autopilot, International Journal of Applied Mathematics and Computer Science 20(2): 271-280, DOI: 10.2478/v10006-010-0020-8.

Velagic, J., Vukic, Z. and Omerdic, E. (2003). Adaptive fuzzy ship autopilot for track-keeping, Control Engineering Practice 11(4): 433-443.

Witkowska, A. and Smierzchalski, R. (2009). Nonlinear backstepping ship course controller, International Journal of Automation and Computing 6(3): 277-284.

Witkowska, A., Tomera, M. and Śmierzchalski, R. (2007). A backstepping approach to ship course control, International Journal of Applied Mathematics and Computer Science 17(1): 73-85, DOI: 10.2478/v10006-007-0007-2.

Zhang, X.K. and Jia, X.L. (2000). Robust PID algorithm based on closed-loop gain shaping and its application on level control, Shipbuilding of China 41(3): 35-39.

Zwierzewicz, Z. (2004). On the adaptive ship track-keeping system design via LQR-integral control, Proceedings of the 10th IEEE International Conference on Methods and Models in Automation and Robotics, MMAR, Międzyzdroje, Poland, pp. 207-212.

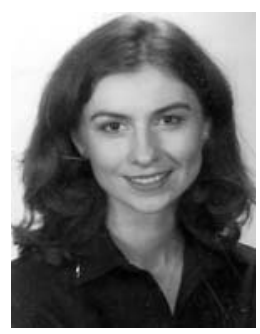

Anna Witkowska holds an M.Sc. in mathematics and computer science from the University of Gdańsk, Poland (2001) and a Ph.D. in automatic control and robotics (2011) from the Technical University of Warsaw. Her research interests include automation, especially control of nonlinear systems such as ocean vehicles. 


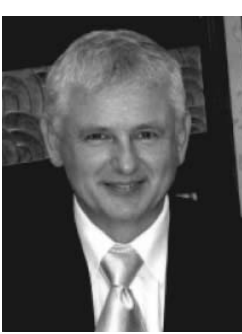

Roman Śmierzchalski graduated in 1979 from

the Technical University of Gdańsk in Poland, Electrical Department, specializing in ship automatics. He received the $\mathrm{Ph} . \mathrm{D}$. degree from the Technical University of Gdańsk, Shipbuilding Institute, in 1988, and the D.Sc. (habilitation) degree in automation and robotics from the Technical University of Warsaw, Faculty of Electronic Engineering, in 1999. Currently he is a professor of the Gdańsk University of Technology, Faculty of Electrical and Control Engineering. He specializes in the development of computer methods of safe control of ship motion. He has published about 150 papers in refereed journal and conference. His current research interests are in the field of artificial intelligent in ship control and automation.

Received: 16 June 2011

Revised: 17 February 2012 